\title{
Ácaros (Acari, Arachnida) associados a euforbiáceas nativas em áreas de cultivo de seringueiras (Hevea brasiliensis Muell. Arg., Euphorbiaceae) na região noroeste do estado de São Paulo, Brasil ${ }^{1}$
}

\author{
Reinaldo J.F. Feres ${ }^{2}$ \\ Maria Andréia Nunes 2,3
}

\begin{abstract}
Mites (Acari, Arachnida) associated with weed Euphorbiaceae in monoculture planting of the amazonian rubber tree (Hevea brasiliensis Muell. Arg., Euphorbiaceae) in Northwestern São Paulo State, Brazil. This paper reports twenty mite species belonging to eighteen genera in nine families, associated with three species of euphorbiaceous weed species: Chamaesyce hirta (Linnaeus) Millsp., Euphorbia heterophylla L. and Phyllanthus tenellus (Muell. Arg.) Roxb., in three different monoculture areas of Hevea brasiliensis. The largest richness of mite species was observed on $C$. hirta, with nine species. The most abundant species were Pronematus sp. and Polyphagotarsonemus latus (Banks, 1904), collected on C. hirta and E. heterophylla.
\end{abstract}

KEY WORDS. Acari, mites, weed Euphorbiaceae, biodiversity, Brazil

Muitas espécies de ácaros são importantes pragas agrícolas (YANINEK \& MORAES 1991), enquanto outras são importantes inimigos naturais de pragas (MORAES et al. 1986). O estudo de ácaros fitófagos e seus inimigos naturais pode tornar possível um melhor manejo das espécies pragas, através da alteração proposital de componentes bióticos de agrossistemas e sistemas adjacentes, visando promover a ação de inimigos naturais nativos.

A acarofauna de seringueiras é bastante diversificada, e está representada atualmente por 29 espécies, das quais pelo menos 13 são fitófagas e 10 predadoras; outras seis são saprófagas, ficófagas ou micófagas, ou ainda de hábito alimentar não bem definido. Três das espécies fitófagas, Calacarus heveae, Oligonychus gossypii e Tenuipalpus heveae tem causado desfolhamentos e grandes infestações de $C$. heveae tem causado perdas na produção de látex (FERES 2000).

O conhecimento da acarofauna associada a plantas nativas é básico para futuros estudos de manejo de agroecossistemas, pois essas plantas podem servir como hospedeiros alternativos para ácaros fitófagos e, além disso, pode levar à descoberta de novas espécies de inimigos naturais que poderiam se tornar disponíveis para uso prático.

1) Parte do Programa BIOTA/FAPESP - O Instituto Virtual da Biodiversidade www.biotasp.org.br

2) Departamento de Zoologia e Botânica, Universidade Estadual Paulista. Rua Cristóvão Colombo 2265, Jardim Nazareth, 15054-000 São José do Rio Preto, São Paulo, Brasil. E-mail: reinaldo@zoo.ibilce.unesp.br

3) Bolsista de Iniciação Científica Fapesp. 
O objetivo do presente estudo foi obter informações sobre a biodiversidade de ácaros associados a três espécies de euforbiáceas herbáceas nativas, Chamaesyce hirta (Linnaeus) Millsp., Euphorbia heterophylla L. e Phyllanthus tenellus (Muell. Arg.) Roxb., em três diferentes áreas de cultivo de seringueira (Hevea brasiliensis) na região noroeste do Estado de São Paulo: Cedral (20 55'27'S, 49¹6'52'W), Pindorama ( $\left.21^{\circ} 13^{\prime} 41^{\prime \prime} \mathrm{S}, 48^{\circ} 54^{\prime} 53^{\prime \prime} \mathrm{W}\right)$ e Taquaritinga ( $\left.21^{\circ} 26^{\prime} 35^{\prime \prime} \mathrm{S}, 48^{\circ} 37^{\prime} 58^{\prime \prime} \mathrm{W}\right)$. Foram realizadas coletas trimestrais, de janeiro a outubro de 2000, em meses correspondentes ao início (abril) e meio (julho) do período seco, e início (outubro) e meio (janeiro) do período chuvoso. Foram amostradas plantas em número suficiente para atingir um mínimo de 60 folíolos por espécie estudada, em cada uma das áreas analisadas. As plantas foram acondicionadas separadamente em sacos plásti$\cos$, que foram mantidos em caixas isotérmicas de isopor com gelo- $\mathrm{X}^{\circledR}$ e em laboratório, em refrigerador a cerca de $10^{\circ} \mathrm{C}$ por no máximo uma semana. Os folíolos foram examinados sob microscópio estereoscópico e todos os ácaros encontrados foram capturados com auxílio de estilete e montados em lâminas de microscopia, em meio de Hoyer (JEPPSON et. al. 1975), com exceção dos Oribatida, que foram fixados em álcool etílico a 70\%, clarificados em ácido láctico e estudados em lâmina escavada em preparação temporária (BALOGH 1972). Os ácaros foram identificados e contados sob microscópio com contraste de fase.

A nomenclatura adotada para as categorias superiores é aquela proposta por WoOLLEY (1988). O material estudado está depositado na coleção de Acari (DZSJRP), do Departamento de Zoologia e Botânica, Universidade Estadual Paulista (UNESP), São José do Rio Preto, São Paulo.

O estudo taxonômico dos exemplares associados a essas plantas revelou a ocorrência de 20 espécies pertencentes a 18 gêneros de nove famílias.

\section{Gamasida}

Phytoseiidae Berlese, 1916

\section{Amblyseius chiapensis DeLeon, 1961}

Amblyseius chiapensis DeLeon, 1961a: 85. - DeLeon, 1962: 12. - McMurtry, 1983: 250.

Material examinado. BRAsIL, São Paulo: Taquaritinga, 12 fêmeas e 4 machos 14-IV-2000, 1 fêmea 21/VII/2000, C. hirta.

Distribuição. Brasil (São Paulo), Colômbia, El Salvador, Guatemala, México, Porto Rico (MORAES et al. 1986).

Observações. Ácaros desta família alimentam-se de outros ácaros, principalmente daqueles pertencentes à família Tetranychidae, e de pólen (FLECHTMANN \& MCMURTRY 1992a; b). Esta espécie apresenta coloração amarelada translúcida em vida. Foram coletados sobre ramos e inflorescências.

\section{Euseius citrifolius Denmark \& Muma, 1970}

Euseius citrifolius Denmark \& Muma, 1970: 222. - Moraes \& McMurtry, 1983: 138. - Feres \& Moraes, 1998: 125. - Feres \& Flechtmann, 1995: 534. - Feres, 2000: 161.

Material examinado. BRAsiL, São Paulo: Cedral, 1 fêmea e 2 machos 14-IV2000, E. heterophylla; Pindorama, 1 fêmea 14/IV/2000, E. heterophylla; Taquaritinga,

Revta bras. Zool. 18 (4): 1253 - 1264, 2001 
6 fêmeas e 2 machos, 14-IV-2000, E. heterophylla; Pindorama, 1 fêmea 21-VII-2000, C. hirta; Taquaritinga, 5 fêmeas e 4 machos 14-IV-2000, C. hirta.

Distribuição. Brasil (Bahia, Ceará, Paraíba, Pernambuco, São Paulo), Paraguai, Peru (MORAES et al. 1986), Colômbia e Nicarágua (MORAES et al. 1991).

Observações. Esta espécie de fitoseídeo ocorre sobre diversas espécies de plantas. Na região noroeste do Estado de São Paulo é a mais freqüente e abundante (FERES \& MORAES 1998). Apresentam coloração branca translúcida em vida. Foram coletados preponderantemente na página inferior dos folíolos, junto da nervura central. Em C. hirta foram coletados principalmente sobre as inflorescências.

\section{Euseius concordis (Chant, 1959)}

Typhlodromus (Amblyseius) concordis Chant, 1959: 69.

Amblyseius (Iphiseius) concordis; Muma, 1961: 288.

Amblyseius concordis; Chant \& Baker, 1965: 22. - Moraes \& McMurtry, 1983: 138.

Euseius concordis; Denmark \& Muma, 1973: 264. - Moraes \& Oliveira, 1982: 317. - Moraes \& McMurtry, 1983: 138. - Feres \& Moraes, 1998: 127. - Feres, 2000: 161

Euseius flechtmanni Denmark \& Muma, 1970: 223. - Denmark \& Muma, 1973: 261 (sinônimo júnior de acordo com Moraes et al., 1982: 18).

Material examinado. Brasil, São Paulo: Cedral, 1 fêmea 26-I-2000, E. heterophylla.

Distribuição. Argentina, Brasil (Bahia, Ceará, Paraíba, Pernambuco, São Paulo), Colômbia, Costa Rica, El Salvador, Guatemala, Iugoslávia, Nicarágua; Portugal e Trinidad-Tobago (MORAES et al. 1986).

\section{Iphiseiodes zuluagai Denmark \& Muma, 1972}

Iphiseiodes zuluagai Denmark \& Muma, 1972: 23. - Denmark \& Muma, 1973: 251. - Denmark \& Muma, 1975: 287. - Moraes et al., 1982: 18. - Aponte \& McMurtry, 1995: 176.

Amblyseius zuluagai; Moraes et al., 1982: 18. - Aponte \& McMurtry, 1995: 176. - Feres \& Flechtmann, 1995: 534 .

Iphiseiodes quadripilis Aponte \& McMurtry, 1993: 151.

Material examinado. BRASIL, São Paulo: Taquaritinga, 2 machos 14-IV-2000, 25 fêmeas e 2 machos 21-VII-2000, C. hirta; 2 fêmeas 14/04/2000, E. heterophylla.

Distribuição. Brasil (São Paulo), Colômbia, Cuba, Porto Rico e Panamá (MORAES et al. 1991).

Observações. Apresentam coloração marrom-avermelhada em vida. Foram coletados na página inferior dos folíolos, junto da nervura central. Em C. hirta foram encontrados sobre as inflorescências.

\section{Typhlodromalus aripo DeLeon, 1967}

Typhlodromalus aripo; DeLeon, 1967:21. - Denmark \& Muma, 1973: 258.

Amblyseius aripo; Moraes \& McMurtry, 1983: 132. - Moraes \& Mesa, 1988: 73. - Feres \& Moraes, 1998: 126.

Material examinado. BRASIL, São Paulo: Taquaritinga, 6 fêmeas 26-I-2000, C. hirta.

Distribuição. Brasil (Bahia, Pernambuco, São Paulo), Colômbia, El Salvador, Guiana, Jamaica e Trinidad (Moraes \& MESA 1988). 


\section{Typhlodromus annectens DeLeon,1958}

Typhlodromus annectens DeLeon, 1958: 75. - Chant \& Yoshida-Saul, 1984: 1868. - Moraes \& McMurtry, 1983: 142. - Moraes \& Mesa, 1988: 82. - Moraes et al., 1991: 134. - Feres \& Moraes, 1998: 128. - Feres, 2000: 161 .

Galendromus annectens; Muma, 1961: 298. - Muma, 1963: 20. - Muma et al., 1970: 135. - Denmark \& Muma, 1973: 274. - Farias et al., 1981: 316. - Moraes et al., 1982: 21.

Material examinado. BRASIL, São Paulo: Pindorama, 1 fêmea 14-IV-2000, C. hirta.

Distribuição. Brasil (Pernambuco, São Paulo), Canadá, Colômbia, E.U.A., Galápagos, Honduras, Jamaica, México, Porto Rico (Moraes et al. 1986).

\section{Typhlodromus sp.}

C. hirta.

Material examinado. BrasiL, São Paulo: Pindorama, 1 fêmea 14-IV-2000,

\section{Actinedida}

Cheyletidae Leach, 1815

\section{Cheletomimus duosetosus Muma, 1964}

Cheletomimus duosetosus Muma, 1964: 242.

Material examinado. BRASIL, São Paulo: Taquaritinga, 1 fêmea 21-VII2000, C. hirta.

Distribuição. Brasil (São Paulo) (FerES \& FleChtMAnN 1995); E.U.A. (MUMA 1964).

Observações. Ácaros de coloração amarelada. Distinguem-se pelo grande desenvolvimento do palpo, cujo fêmur é o maior segmento e apresenta-se curvado como cotovelo na posição mediana. A maioria das espécies é predadora de grande variedade de pequenos artrópodes, como ácaros e Collembola. Algumas espécies ocorrem sobre a folhagem das plantas onde se alimentam de ácaros fitófagos e cochonilhas (FLECHTMANN 1975).

\section{Stigmaeidae Oudemans, 1931}

\section{Zetzellia sp.}

Material examinado. BRASIL, São Paulo: Pindorama, 1 fêmea 14-IV-2000, C. hirta.

Distribuição. Gênero com espécies de ampla distribuição geográfica (WOOD 1967).

Observações. É conhecido o hábito alimentar de Z. mali (Ewing), predador de várias espécies de ácaros tetraniquídeos na América do Norte, Europa e Israel (JEPPSON et al. 1975). 


\section{Tydeidae Kramer 1877}

\section{Lorryia formosa Cooreman, 1958}

Lorryia formosa Cooreman, 1958: 6-10. - Baker, 1968: 995-996. - Feres \& Flechtmann, 1995: 535.

Material examinado. Brasil, São Paulo: Taquaritinga, 1 fêmea 21-VII2000, C. hirta.

Distribuição. Argentina, Brasil, Equador, Espanha, França, Marrocos, México, Uruguai (BAKER 1968), Paraguai (FleCHTMAnN 1973).

Observações. Ácaros de coloração verde-amarelada. Espécie não identificada deste gênero foi registrada em seringueiras no Município de Rio Claro, São Paulo, por FLECHTMANN \& ARLEU (1984). Ocorrem ao longo do ano, sobre um grande número de plantas, sem causar dano aparente às folhas.

\section{Pronematus sp.}

Material examinado. Brasil, São Paulo: Cedral, 4 fêmeas e 1 macho 26-I-2000, 4 fêmea e 1 macho 14/IV/2000, 1 macho 20-X-2000, E. heterophylla, 1 fêmea 21-VII-2000, C. hirta; Pindorama, 22 fêmeas e 2 machos 14-IV-2000, 2 fêmeas 21-VII-2000, 4 fêmeas 20-X-2000, C. hirta; Taquaritinga, 3 fêmeas e 1 macho 21-VII-2000, 1 fêmea 20-X-2000, C. hirta.

Observações. São diminutos, ranslúcidos e levemente brancacentos e de movimentos rápidos. São referidos como predadores de eriofiídeos, embora existam dúvidas a respeito do hábito alimentar das espécies pertencentes a este gênero e gêneros afins (BAKER \& WHARTON 1952; BAKER 1965; MCCOY et al. 1967; CALVERT \& HUFFAKER 1974; BAYAN 1986).

\section{Parapronematus sp.}

Material examinado. Brasil, São Paulo: Pindorama, 1 fêmea 14-IV-2000, C. hirta.

Observações. Semelhantes a Pronematus sp. na forma e coloração, diferindo deste por apresentarem setas bífidas sobre os fêmures III e IV.

\section{Tarsonemidae Kramer, 1877}

\section{Polyphagotarsonemus latus (Banks, 1904).}

Tarsonemus latus Banks, 1904: 1553.

Hemitarsonemus latus; Ewing, 1939: 54.

Neotarsonemus latus; Smiley, 1967: 137.

Polyphagotarsonemus latus; Beer \& Nucifora, 1965: 38-42. - Feres, 2000: 164.

Material examinado. Brasil, São Paulo: Pindorama, 10 fêmeas e 1 macho 14-IV-2000, C. hirta; Taquaritinga, 11 fêmeas e 3 machos 14-IV-2000, 14 fêmeas e 2 machos 20-X-2000, C. hirta, 2 fêmeas e 1 macho 20-X-2000, E. heterophylla.

Distribuição. Ampla distribuição mundial, ocorrendo em países de regiões tropicais e temperadas quentes (LINDQUIST 1986). 
Observações. Espécie conhecida como ácaro branco ou tropical e praga de diversas culturas. Prefere clima quente e úmido (FleChTMANN 1975). A coloração varia de esbranquiçada ao amarelo escuro. Em C. hirta foram coletados na página inferior dos folíolos e sobre as inflorescências.

\section{Tenuipalpidae Berlese,1913}

\section{Brevipalpus phoenicis (Geijskes, 1939)}

Tenuipalpus phoenicis Geijskes, 1939: 23.

Brevipalpus phoenicis; Sayed, 1946. - Pritchard e Baker, 1958: 233. - DeLeon, 1961: 48. - Gonzales,

1975: 82. - Baker et al., 1975: 18. - Meyer, 1979: 87. - Baker \& Tuttle, 1987: 98- 99.

Brevipalpus yothersi Baker, 1949: 374.

Brevipalpus mcbridei Baker, 1949: 374.

Brevipalpus papayensis Baker, 1949: 379.

Material examinado. BRASIL, São Paulo: Cedral, 1 fêmea 14-IV-2000, E. heterophylla.

Distribuição. Espécie de ampla distribuição geográfica, ocorrendo sobre grande número de espécies de plantas hospedeiras. África (MEYER \& RODRIGUES 1965; Rodrigues 1968; MeYER 1979), Brasil (Alagoas, Bahia, Cará, Minas Gerais, Paraná, Pernambuco, Rio de Janeiro, São Paulo) (Flechtmann 1976), Colômbia (Zuluaga \& Saldarriaga 1970), E.U.A. (BAKer \& Suigong 1988), México (BAKER \& TUTTLE 1987), Porto Rico (COMROY 1958).

Observações. Referido como "ácaro da leprose dos citros", pois é vetor da virose que causa essa patogenia nos citros (CHIAVEGATO 1980). Já foi registrado em seringueiras de Itabuna, Bahia e Rio Claro, São Paulo (FLECHTMAnN \& ARLEU 1984) e de outros municípios do Estado de São Paulo (FERES 2000).

\section{Tenuipalpus heveae Baker, 1945}

Tenuipalpus heveae Baker, 1945: 36. - Baker \& Prichard, 1953: 320. - Feres, 2000: 165.

Material examinado. BRASIL, São Paulo: Pindorama, 4 fêmea 14-IV-2000, E. heterophylla.

Distribuição. Brasil: Amazonas (Flechtmann \& ARLEU 1984), Pará (BAKER 1945), São Paulo (FERES 2000).

Observações. Ácaro plano, conhecido no estado do Pará como ácaro vermelho da seringueira (FLECHTMANN 1979). Ocorrem predominantemente na face inferior das folhas medianas e mais velhas da seringueiras. Quando em grande infestação, também são encontrados na face superior. Foram registradas grandes infestações de ácaros dessa espécie em seringais de Barretos, São Paulo, durante o mês de fevereiro, e em Goianésia, Gioás, durante o mês de outubro (J.F.C. Benesi com. pess.; R.J.F. Feres obs. pess.). 


\section{Tetranychidae Donnadieu, 1875 \\ Eutetranychus banksi (McGregor, 1914)}

Tetranychus banksi McGregor, 1914: 358.

Anychus banksi; McGregor, 1919: 644.

Eutetranychus banksi; McGregor, 1950: 268. - Prichard \& Baker, 1955: 115. - Flechtmann \& Baker,

1970: 156. - Flechtmann \& Baker, 1975:112. - Feres, 2000: 165.

Material examinado. BRAsil, São Paulo: Cedral, 2 ninfas 14-IV-2000, E. heterophylla.

Distribuição. Argentina, Brasil, Colômbia, Costa Rica, Cuba, Equador, El Salvador, E.U.A., Guatemala, Havaí, Honduras, México, Nicarágua, Panamá, Paraguai, Peru, Uruguai, Venezuela (Bolland et al. 1998).

Observações. Nos Estados Unidos da América do Norte esta espécie é considerada séria praga dos citros (CHIAVEGATO 1980). Ocorre sobre grande número de plantas (BOLLAND et al. 1998).

\section{Oligonychus gossypii (Zacher, 1920)}

Paratetranychus gossypii Zacher, 1920: 183.

Oligonychus gossypii; Pritchard \& Baker, 1955: 359. - Baker \& Pritchard, 1960: 508. - Meyer, 1974: 263. - Meyer, 1987: 152.

Material examinado. BRASIL, São Paulo: Cedral, 1 macho e 2 ninfas 14-IV2000, E. heterophylla; Pindorama, 3 ninfas 14-IV-2000, E. heterophylla; Taquaritinga, 1 fêmea 21-VII-2000, P. tenellus.

Distribuição. Angola, Benin, Brasil, Camarões, Colômbia, Congo, Costa Rica, Equador, Etiópia, Honduras, Madagascar, Nigéria, Quênia, República Centro-Africana, São Tomé, Senegal, Serra Leoa, Tanzânia, Togo, Uganda, Venezuela, Zaire (BOLLAND et al. 1998).

Observações. Registrada em vários países da África e nas Américas Central e do Sul, em várias espécies de plantas. Registrada pela primeira vez em seringueiras cultivadas em 1986 e 1988, nos estados do Mato Grosso, Acre e Amazonas, causando desfolhamentos (FAZOLIN \& PEREIRA 1989; FleCHTMANN 1989). Habitam a superfície inferior das folhas, onde produzem teias.

\section{Mononychellus sp.}

Material examinado. Brasil, São Paulo: Cedral, 1 macho 14-IV-2000, E. heterophylla.

\section{Astigmata \\ Winterschmidtiidae Oudemans, 1923}

\section{Oulenzia sp.}

Material examinado. BRASIL, São Paulo: Taquaritinga, 7 fêmeas e 2 machos 21-VII-2000, C. hirta. 
Observações. Ácaros de coloração brancacenta brilhante. O. arboricola (Oudemans) foi descrita sobre folhas de Hevea em Sumatra; espécimes também foram coletados sobre juta, na Índia (BAKER \& WHARTON 1952), sendo considerados de hábito herbívoro ou micófago (BAKER \& WHARTON 1952, KRANTZ 1978, WOOLEY 1988).

\section{Oribatida}

\section{Mochlozetidae Grandjean, 1960}

Material examinado. BRASIL, São Paulo: Taquaritinga, 5 fêmeas e 1 macho 14-IV-2000, E. heterophylla.

Observações. Espécimes pertencentes a essa família de Oribatida foram encontrados sobre flores e frutos de E. heterophylla. Outros três exemplares imaturos de oribatídeos também foram coletados nessa mesma data, no mesmo local e em Pindorama, sobre flores e frutos de $C$. hirta.

Considerando-se a acarofauna das plantas estudadas, a maior riqueza de espécies (15) e a maior densidade ( 0,7 ácaro/folíolo), ocorreram no período seco do ano. No período chuvoso registrou-se uma diminuição sensível tanto da riqueza de espécies (3), como da densidade (0,04 ácaro/folíolo).

Dentre as três espécies de euforbiáceas herbáceas nativas estudadas, Chamaesyce hirta foi a que apresentou a maior riqueza de espécies acarinas (13 espécies, $65 \%$ da acarofauna total), enquanto $P$. tenellus apresentou a menor riqueza (uma única espécie). Algumas das 20 espécies acarinas registradas nessas euforbiáceas nativas estudadas, foram comuns entre elas. Em Chamaesyce hirta e Euphorbia heterophylla foram registradas 19 espécies, das quais quatro (20\%) foram comuns às duas plantas. Em $E$. heterophylla e $P$. tenellus foram registradas 11 espécies, sendo apenas uma comum às duas plantas. Nenhuma espécie foi comum a $C$. hirta e P. tenellus.

Todas as espécies acarinas coletadas sobre as três euforbiáceas nativas estudadas são registradas pela primeira vez nessas plantas, com exceção de $E$. concordis, já registrada em $E$. heterophylla $(=E$. geniculata $)$. Para $C$. hirta, existe o registro de Typhlodromus gutierrezi em Madagascar (MORAES et al. 1986), espécie não registrada neste trabalho.

A acarofauna das euforbiáceas nativas foi composta por um maior número de espécies preponderantemente predadoras (11) que de fitófagas (8). Cinco espécies foram abundantes, das quais quatro são preponderantemente predadoras (Iphiseiodes zuluagai, Euseius citrifolius, Amblyseius chiapensis e Pronematus sp.) e uma fitófaga (Polyphagotarsonemus latus).

Das 20 espécies registradas nessas euforbiáceas nativas, apenas seis não foram registradas em seringueiras: Amblyseius chiapensis, Cheyletomimus duosetosus, Mononychellus sp., Typhlodromalus aripo, Typhlodromus sp. e uma espécie de Mochlozetidae (Oribatida).

Os fitoseídeos E. citrifolius e I. zuluagai, ocorreram em diferentes microhábitats dependendo da planta hospedeira: em $C$. hirta, foram registrados em inflorescências e em $E$. heterophylla, embora florida, foram registrados somente na página 
inferior dos folíolos. Esse fato pode ser determinado por uma possível preferência peỉo pólen produzido pelas flores de $C$. hirta, já que este é fonte alternativa de alimento para ácaros dessa família.

Nenhuma das espécies de eriofiídeos de Hevea brasiliensis (Calacarus heveae Feres, 1992, Phyllocoptruta seringueirae Feres 1998 e Shevtchenkella petiolula Feres, 1998) foi registrada nessas euforbiáceas nativas estudadas.

\begin{abstract}
AGRADECIMENTOS. Às Dras Denise de C. Rossa Feres e Neusa T. Ranga, Departamento de Zoologia e Botânica, UNESP, São José do Rio Preto, pela leitura crítica do manuscrito e sugestões apresentadas, e pela identificação das plantas analisadas, respectivamente; ao biólogo Anibal Ramadan Oliveira, doutorando do Curso de Pós-Graduação em Ciências - Zoologia, Departamento de Zoologia, Instituto de Biociências, USP, pela identificação dos Oribatida; ao Engenheiro Agrônomo José Fernando Canuto Benesi, Diretor Técnico da Associação Paulista dos Produtores e Beneficiadores de Borracha (APABOR), pelas informações prestadas sobre infestações de Tenuipalpus heveae em Barretos, São Paulo e Goianésia, Goiás; à FAPESP, pelo apoio financeiro (Processos n. 98/7099-0 e 99/06898-0).
\end{abstract}

\title{
REFERÊNCIAS BIBLIOGRÁFICAS
}

Aponte, O. \& J.A. McMurtry. 1993. Phytoseiid mites of Venezuela (Acarina: Phytoseiidae). Internat. Jour. Acarol. 12: 149-157.

- 1995. Revision of the genus Iphiseiodes De Leon (Acari: Phytoseiidae). Internat. Jour. Acarol. 21 (3): 165-183.

BAKER, E.W. 1945. Mites of genus Tenuipalpus (Acarina: Trichadenidae). Proc. Entomol. Soc. Wash. 47 (2): 33-44.

- 1949. The genus Brevipalpus (Acarina: Pseudoleptidae). Am. Midl. Nat. 42 (2): 350-402.

. 1965. A review of the genera of the Family Tydeidae (acarina). Adv. Acarol. 2: 95-133.

. 1968. The genus Lorryia. Ann. Entomol. Soc. Amer. 61 (4): 986-1008.

BAKER, E.W. \& A.E. PRITCHARD. 1953. A review of the false spider mite genus Tenuipalpus Donnadieu (Acarina; Phytoptipalpidae). Ann. Entomol. Soc. Amer. 46 (3): 317-336.

. 1960. The Tetranychoid mites of Africa. Hilgardia 29 (11): 455-574.

BAKER, E.W. \& Y. Suigong. 1988. A Catalog of the false spider mites (Tenuipalpidae: Acari) of the United States. Internat. Jour. Acarol. 14 (3): 143-155.

BAKER, E.W. \& D.M. TUTTLE. 1987. The False spider mite of Mexico (Tenuipalpidae: Acari). Tech.

Bull. U.S. Dept. Agric. (1706): 1-237.

BAKeR, E.W., D.M. TUTTLE \& M.J. ABATIELlo. 1975. The False spider mite of northwestern and north central Mexico (Acarina: Tenuipalpidae). Smithsonian Contrib. Zool. 194: 1-23.

Baker, E.W. \& G.W. Wharton. 1952. An introduction to Acarology. New York, MacMillan Co., $465 \mathrm{p}$

BALOGH, J. 1972. The oribatid genera of the world. Budapest, Akadémiai Kiadó, 188p.

Banks, N. 1904. Class III, Arachnida, Order I, Acarina, four new species of injurious mites. Jour. N.Y. Entomol. Soc. 12: 53-56.

BAYAN, A. 1986. Tydeid mites associated with aplles in Lebanon (Acari: Actinedida: Tydeidae). Acarologia 27 (4): 311-316.

BEER, R.E. \& A. NuCIFORA. 1965. Revisione dei generi della famiglia Tarsonemidae (Acarina). Bol. Zool. Agric. Bachic. (2) 7: 19-43.

Bolland, H.R., J. Gutierrez \& C.H.W. Flechtmann. 1998. World catalog of the spider mite family (Acari: Tetranychidae). Leiden, Brill, 392p.

Calvert, D.J. \& C.B. Huffaker. 1974. Predator (Metaseiulus occidentalis) prey (Pronematus spp.) 
interactions under sulfur and cattail pollen applications in a noncommercial vineyard. Entomophaga 19 (3): 361-369.

Chant, D.A. 1959. Phitoseiid mites (Acarina: Phytoseiidae). Part I. Binomics of seven species in southeastern England. Part II. A taxonomic review of the family Phytoseiidae, with descriptions of thirty-eight new species. Canad. Entomol. 12 (Suppl.): 1-166.

Chant, D.A. \& E.W. Baker. 1965. The Phytoseiidae (Acarina) of Central America. Mem. Entomol. Soc. Canada 41: 1-56.

Chant, D.A. \& Yoshida-Shaul. 1984. A world review of the occidentalis species group in the genus Typhlodromus Scheuten (Acarina Phytoseiidae). Canad. Jour. Zool. 62: 1860-1871.

Chiavegato, L.G. 1980. Ácaros da cultura dos citros, p. 469-501. In: O. Rodriguez \& F.C.P. ViéGAS (Ed.). Citricultura brasileira. Campinas, Fundação Cargill, 739p.

Comroy, H.L. 1958. Preliminary survey of the plant mites of Puerto Rico. Jour. Agric. Univ. Puerto Rico 42 (2): 39-144.

Cooreman, J. 1958. Notes et observations sur les Acariens. VII-Photia graeca n.sp. (Acaridiae, Canestriniidae) et Lorryia formosa n.sp. (Stomatostigmata, Tydeidae). Bull. Inst. Roy. Sci. Nat. Belgique 34 (8): 1-10.

DeLEON, D. 1958. Four new Typhlodomus from southern Florida (Acarina: Phytoseiidae). Florida Entomol. 41: 73-76.

- 1961a. Eight new Amblyseius from Mexico with collection notes on two other species (Acarina: Phytoseiidae). Florida Entomol. 44 (1): 41-52.

. 1961b. The genus Brevipalpus in Mexico. Part II (Acarina: Tenuipalpidae). Florida Entomol. 44 (2): 85-91.

. 1962. The cervices of some Phytoseiid type specimens (Acarina: Phytoseiidae). Acarologia 4: $174-176$.

. 1967. Some mites of the Caribbean Area. Lawrence, Allen Press Inc., 66p.

Denmark, H.A. \& M.H. Muma. 1970. Some Phytoseiid mites of Paraguay (Phytoseiidae: Acarina). Florida Entomol. 53 (4): 219-227.

. 1972. Some Phytoseiidae of Colombia (Acarina: Phytoseiidae). Florida Entomol. 55 (1): 19-29.

- 1973. Phytoseiidae mites of Brazil (Acarina: Mesostigmata). Rev. Brasil. Biol. 33: 235-276.

1975. The Phytoseiidae (Acarina: Mesostigmata) of Puerto Rico. Jour. Agric. Univ. Puerto Rico 59: 279-304.

EwING, H. E. 1939. A revision of the mites of the subfamily Tarsoneminae of North America, the West Indies and the Hawaiian İslands. Tech. Bull. U.S. Dept. Agric. 653: 1-63.

Farias, A.R.; C.H.W. Flechtmann; G.J. De Moraes \& J.A. McMurtRy. 1981. Predadores do ácaro verde da mandioca, no nordeste do Brasil. Pesq. Agropec. Brasil., Rio de Janeiro, 16 (3): 313-317.

Fazolin, M. \& L.V. Pereira. 1989. Ocorrência de Oligonychus gossypii (Zacher, 1920) (Acari: Tetranychidae) em seringueiras cultivadas. An. Soc. Entomol. Brasil 18 (1): 199-202.

FERES, R.J.F. 1992. A new species of Calacarus Keifer (Acari:Eriophyidae, Phyllocoptinae) from Hevea brasiliensis Muell. Arg. (Euphorbiaceae) from Brazil. Internat. Jour. Acarol. 18 (1): 61-65.

- 1998. Two new Phyllocoptine mites (Acari: Eriophyidae) from Hevea brasiliensis Muell. Arg, (Euphorbiaceae) from Brasil. Internat. Jour. Acarol. 24 (1): 69-74.

. 2000. Levantamento e observações naturalísticas da acarofauna (Acari, Arachnida) de seringueiras cultivadas (Hevea spp., Euphorbiaceae) no Brasil. Revta bras. Zool. 17 (1): 157-173.

FERES, R.J.F. \& C.H.W. Flechtmann. 1995. Mites (Acari) associated with bamboo (Bambusa sp., Poaceae) in a woody area from northwestern São Paulo State, Brazil. Revta bras. Zool. 12 (3): 533-546.

FERES, R.J.F. \& G.J. DE MoRAEs. 1998. Phytoseiid mites (Acari:Phytoseiidae) from woody areas in the State of São Paulo, Brazil. Syst. Appl. Acarol. 3: 125-132.

FleChTMANn, C.H.W. 1973. Lorryia formosa Cooremann, 1958 - Um ácaro dos citros pouco conhecido no Brasil. Ci. Cult. 25 (12): 1179-1181. 
1975. Elementos de Acarologia. São Paulo, Livraria Nobel S.A., 344p.

1976. A report on the Tetranychidae (Acari) of Brazil: An emmendation. Revta bras. Ent.

20 (2): 115-116.

1979. Tuckerella ornata (Tucker), um ácaro novo para o Brasil e outros Tetranychoidea

(Acari) do estado do Pará. An. Esc. Sup. Agric. “Luiz de Queiroz”, Piracicaba, 36: 615-620.

1989. Seringueira (Hevea sp.), um novo hospedeiro para Oligonychus gossypii (Zacher,

1920) (Acari: Tetranychidae). Bol. Mus. Paraense Emílio Goeldi, Sér. Zool., 5 (1): 127-128.

FleChtMAnN, C.H.W. \& R.J. ARLEU. 1984. Oligonychus coffeae (Nietner, 1981), um ácaro tetraniquídeo da seringueira (Hevea brasiliensis) novo para o Brasil e observações sobre outros ácaros desta planta. Ecossistema 9: 123-125.

Flechtmann, C.H.W. \& E.W. Baker. 1970 A. Preliminary report on the Tetranychidae (Acarina) of Brazil. Ann. Entomol. Soc. Amer. 63 (1): 156-163.

1975. A report on the Tetranychidae (Acari) of Brazil. Revta bras. Ent. 19 (3): 111-122.

Flechtmann, C.H.W. \& J.A. McMurtry. 1992a. Studies on how Phytoseiid mites feed on spider mites and pollen. Internat. Jour. Acarol. 18 (3): 157-162.

. 1992b. Studies of cheliceral and deutosternal morphology of some Phytoseiid (Acari: Mesostigmata) by scanning electron microscopy. Internat. Jour. Acarol. 18 (3): 163-169.

GEIJSEES, D.C. 1939. Beiträge zur Kenntnis det europäischen Spinnmilben (Acari, Tetranychidae), mit besonderer Berücksichtigung der niederländischen Arten. Meded. van de Landbouwhoogesch. te Wageningen (Nederland) 42 (4): 1-68.

GonZalez, R.H. 1975. Revision of the Brevipalpus phoenicis "complex", with descriptions of new species from Chile and Thailand (Acarina, Tenuipalpidae). Acarologia 17 (1): 82-91.

JEPPSON, L.R.; H.H. KEIFER \& E.W. BAKER. 1975. Mites injurious to economic plants. Berkeley, Univ. California Press, XXIV+64lp.

Krantz, G.W. 1978. A manual of Acarology. Covalis, Oregon State Univ. Bookstores Inc., 509p.

LINDQUIST, E.E. 1986. The world genera of Tarsonemidae (Acari: Heterostigmata): A morphological, Phylogenetic, and Systematic revision, with a reclassification of family-group taxa in the Heterostigmata. Mem. Entomol. Soc. Canada (36): 1-517.

McCoy, C.W., A.G. Selhime \& R.F. Kanavel. 1967. The feeding behavior and biology of Parapronematus acaciae (Acarina: Tydeidae). Florida Entomol. 52 (1): 13-19.

McGregor, E.A. 1914. Four new tetranychids. Ann. Entomol. Soc. Amer. 7: 354-364.

1919. The red spiders of America a few european species likely to be introduced. Proc. U.S. Nat. Mus. 56: 641-679.

1950. Mites of the family Tetranychidae. Amer. Midl. Nat. 44 (2): 257-420.

MCMurtry, J.A. 1983. Phytoseiid mites from Guatemala, with descriptions of two new species and redefinitions of the genera Euseius, Typhloseiopsis, and Typhlodromus occidentalis species group (Acari: Mesostigmata). Int. Jour. Entomol. 25: 249-272.

MEYER, M.K.P.S. 1974. A revision of the Tetranychidae of Africa (Acari) with a key to the genera of the world. Entomol. Mem. Tech. Serv. Repub. S. Afr. (36): 1-291.

1979. The Tenuipalpidae (Acari) of Africa with keys to the world fauna. Entomol. Mem. Dept. Agric. Tech. Serv. Repub. S. Afr. (50): 1-135.

1987. African Tetranychidae (Acari: Prostigmata), with reference to the world genera. Entomol. Mem. Agric. Wat. Supply. Repub. S. Afr. (69): 1-175.

MEYER, M.K.P.S. \& M. DA C. RodRIGUES. 1965. Acari associated with cotton in Southern Africa (with reference to orther plants). Garcia Orta Lisboa 13 (2): 195-226.

Moraes, G.J. DE \& J.A. MCMUrTRY. 1983. Phytoseiidae mites (Acarina) of Northeastern Brazil. with descriptions of four new species. Internat. Jour. Acarol. 9: 131-148.

Moraes, G.J. DE \& J.V. Oliveira. 1982. Phytoseiid mites of coastal Pernambuco, in northeastern Brazil. Acarologia 23: 315-318.

Moraes, G.J. DE; H.A. Denmark \& J.M. Guerrero. 1982. Phytoseiid mites of Colombia (Acarina, Phytoseiidae). Internat. Jour. Acarol. 8 (1): 15-22. 
Moraes, G.J. De; J.A. McMurtry \& H.A. Denmark. 1986. A Catalog of mite family Phytoseiidae; references to taxonomy, synonymy, distribution and habitat. Brasília, EMBRAPA-DDT, 353p.

Moraes, G.J. DE \& N.C. MesA. 1988. Mites of the family Phytoseiidae (Acari) in Colombia, with descriptions of three new species. Internat. Jour. Acarol. 14 (2): 71-88.

Moraes, G.J. DE; N.C. Mesa \& A. Braun. 1991. Some Phytoseiid mites of Latin America (Acari: Phytoseiidae). Internat. Jour. Acarol. 17 (2): 117-139.

Muma, M.H. 1961. Subfamiles, genera, and species of Phytoseiidae (Acarina: Mesostigmata). Florida St. Mus. Bull. Biol. Sci. 5: 267-302.

- 1963. The genus Galendromus Muma, 1961 (Acarina: Phytoseiidae). Florida Entomol. Suppl. 1: 15-41.

- 1964. Cheyletidae (Acarina: Trombidiformes) associated with citrus in Florida. Florida Entomol. 47 (4): 239-253.

Muma, M.H.; H.A. Denmark \& D. Deleon. 1970. Phytoseiidae of Florida. Arthropods of Florida and neighboring land areas. Gainesville, Florida Dept. Agr. Cons. Serv., Div. Plant Ind., Vol. 6, $150 \mathrm{p}$.

Pritchard, A.E. \& E.W. BaKer. 1955. A revision of the spider mites family Tetranychidae. San Francisco, Pacific Coast Entomological Society, Memoirs series, Vol. 2, 472p.

- 1958. The false spider mites (Acarina: Tenuipalpidae). Univ. Calif. Publ. Entomol. 14 (3): 175-274.

Rodrigues, M. DA C. 1968. Acarina de Moçambique - Catálogo das espécies relacionadas com a agricultura. Agron. moçamb. 2 (4): 215-256.

SAYED, M.T. 1946. Description of Tenuipalpus granati nov. spec. and Brevipalpus pyri nov. spec. $\mathbb{1}^{\mathrm{er}}$ Ent. Bull. Soc. Fouad. $1^{\text {er }}$ 30: 99-104.

Smiley, R.L. 1967. Some Tarsonemidae from the Republic of the Congo (Acarina). Proc. Entomol. Soc. Wash. 66: 145-150.

Wood, T.G. 1967. New Zeland mites of the family Stigmaeidae (Acari, Prostigmata). Trans. Roy. Soc. New Zealand, Zool., 9 (9): 93-139.

Woolley, T.A. 1988. Acarology: mites and human welfare. New York, Wiley-Interscience, 484p.

YANINEK, J.S. \& G.J. DE MoRAES. 1991. Mites in biological and integrated control of pests in agriculture, p. 133-149. In: F. Dusbabek \& V. Bukva (Eds). Modern Acarology. The Hague, Acad. Prague and SPB Academic Publ., Vol. 1, 680p.

ZACHER, F. 1920. Neue und wenig bekannte Spinnmilben. Ztschr. ang. Entomol. 7: 181-187.

ZULUAGA, C. \& V. SALDARRIAGA. 1970. Reconocimiento, identificacion y algunas observaciones sobre dinamica de poblaciones de acaros, en citricos del valle del Cauca. Acta Agron. 20 (3/4): 114-141.

Recebido em 08.III.2001; aceito em 26.X.2001. 